\title{
Mémoire sur la théorie de l'élasticité des corps homogènes à élasticité constante.
}

(Par M. L. Lorenz à Copenhague.)

Nous désignons par $N_{1}, N_{2}, N_{3}$ et $T_{1}, T_{2}, T_{3}$ les composantes normales et tangentielles, rapportées à l'unité de surface, de la force élastique exercée sur les trois plans perpendiculaires aux coordonnées rectangulaires $x, y$ et $z$; par $u, v, w$ les projections sur les axes coordonnés du déplacement d'un point matériel $(x, y, z)$; par $\varrho$ la densité du milieu ; par $\boldsymbol{X}, \boldsymbol{Y}, \boldsymbol{Z}$ les composantes des forces accélératrices qui pourraient agir sur l'élément de la masse.

Les équations qui servent comme point de départ dans les problèmes que je me propose de résoudre, donnent pour les forces élastiques les valeurs

$$
\begin{array}{ll}
\boldsymbol{N}_{1}=\lambda \theta+2 \mu \frac{d u}{d x}, & \boldsymbol{T}_{1}=\mu\left(\frac{d v}{d z}+\frac{d w}{d y}\right), \\
\boldsymbol{N}_{2}=\lambda \theta+2 \mu \frac{d v}{d y}, & \boldsymbol{T}_{2}=\mu\left(\frac{d w}{d x}+\frac{d u}{d z}\right), \\
\boldsymbol{N}_{3}=\lambda \theta+2 \mu \frac{d w}{d z}, & \boldsymbol{T}_{3}=\mu\left(\frac{d u}{d y}+\frac{d v}{d x}\right),
\end{array}
$$

dans lesquelles $\lambda$ et $\mu$ sont des constantes, et $\theta$ c. à. d. la dilatation est exprimée par:

$$
\theta=\frac{d u}{d x}+\frac{d v}{d y}+\frac{d w}{d z}
$$

De ces six équations on déduit, en mettant

$$
\triangle^{2}=\frac{d^{2}}{d x^{2}}+\frac{d^{2}}{d y^{2}}+\frac{d^{2}}{d z^{2}},
$$

dans le cas de l'équilibre d'élasticité:

Journal für Mathematik Bd. LVIII. Heft 4.

$$
\text { (1.) }\left\{\begin{array}{l}
(\lambda+\mu) \frac{d \theta}{d x}+\mu \Delta^{2} u+\varrho X=0, \\
(\lambda+\mu) \frac{d \theta}{d y}+\mu \Delta^{2} v+\varrho \boldsymbol{Y}=0, \\
(\lambda+\mu) \frac{d \theta}{d z}+\mu \Delta^{2} v+\rho \mathbf{Z}=0,
\end{array}\right.
$$


et dans le cas du mouvement:

$$
\left\{\begin{array}{l}
(\lambda+\mu) \frac{d \theta}{d x}+\mu \Delta^{2} u+\rho X=\rho \frac{d^{2} u}{d t^{2}} \\
(\lambda+\mu) \frac{d \theta}{d y}+\mu \Delta^{2} v+\rho Y=\rho \frac{d^{2} v}{d t^{2}} \\
(\lambda+\mu) \frac{d \theta}{d z}+\mu \Delta^{2} w+\rho \mathbb{Z}=\rho \frac{d^{2} w}{d t^{2}}
\end{array}\right.
$$

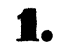

Elimination des forces accélératrices.

Les fonctions $u, v, w$ se composent de deux espèces de termes, dont les uns, que nous désignerons par $u_{0}, v_{0}, w_{0}$ font disparaître les composantes des forces accélératrices $\boldsymbol{X}, \boldsymbol{Y}, \boldsymbol{Z}$; les autres forment les intégrales générales des équations (1.) et (2.), ces forces étant nulles. Nous fểrons donc voir que l'on pourra toujours déterminer les fonctions $u_{0}, v_{0}, w_{0}$, les $X, Y, Z$ étant des fonctions connues quelconques, et pour généraliser le problème nous supposons que ces forces sont aussi des fonctions du temps $t$, ce que nous désignerons en ajoutant un $t$ sous le signe de ces fonctions.

En effectuant les différentiations, on vérifie aisément l'équation

$$
\text { (3.) } \quad a^{2} \triangle^{2} \frac{\varphi\left(t-\frac{r}{a}\right)}{r}=\frac{d^{2}}{d t^{2}} \frac{\varphi\left(t-\frac{r}{a}\right)}{r} \text {, }
$$

où $a$ est une constante, $\varphi(t)$ une fonction quelconque de $t, \alpha, \beta, \gamma$, et $r$ égal à $\sqrt{(x-\alpha)^{2}+(y-\beta)^{2}+(z-\gamma)^{2}}$.

Cela posé considérons l'intégrale

$$
\int d \widetilde{\omega} \frac{\varphi\left(t-\frac{r}{a}\right)}{r}
$$

$d \widetilde{\omega}$ désignant l'élément de volume $d \alpha d \beta d \gamma$ et l'intégration se rapportant à un espace quelconque. En appliquant à cette intégrale les deux opérations $\frac{d^{2}}{d t^{2}}$ et $\Delta^{2}$ on trouve

Mais l'équation

$$
\text { (3.) } \frac{d^{2}}{d t^{2}} \int d \widetilde{\omega} \frac{\varphi\left(t-\frac{r}{a}\right)}{r}=\int d \bar{\omega} \frac{d^{2}}{d t^{2}} \frac{\varphi\left(t-\frac{r}{a}\right)}{r} \text {. }
$$

$$
\text { (3 } \left.{ }^{b} .\right) \quad \Delta^{2} \int d \omega \frac{\varphi\left(t-\frac{r}{a}\right)}{r}=\int d \omega \Delta^{2} \frac{\varphi\left(t-\frac{r}{a}\right)}{r}
$$


n'est exacte que dans le cas où le point $(x, y, z)$ se trouve en dehors des limites de l'intégration relative à $\alpha, \beta, \gamma$. Dans le cas contraire il existe des éléments infinis sous l'intégrale, $r$ pouvant s'évanouir, ot la différentiation sous l'intégrale cesse d'être permise. On trouve aisément la modification que l'équation $\left(3^{b}\right.$.) subit alors en considérant l'intégrale

$$
\int d \widetilde{\omega} \frac{\varphi\left(t-\frac{r}{a}\right)-\varphi(t)}{r} .
$$

L'expression sous cette intégrale ne devient plus infinie lorsque $r$ s'évanouit. On a donc

$$
\Delta^{2} \int d \widetilde{\omega} \frac{\varphi\left(t-\frac{r}{a}\right)-\varphi(t)}{r}=\int d \widetilde{\omega} \Delta^{2} \frac{\varphi\left(t-\frac{r}{a}\right)-\varphi(t)}{r}
$$

pour l'un et l'autre des deux cas distingués ci-dessus; et comme $\Delta^{2} \frac{1}{r}$ qui dans la seconde partie de cette équation se trouve sous l'intégrale est identiquement nul, on a

$$
\text { (3c) } \Delta^{2} \int d \widetilde{\omega} \frac{\varphi\left(t-\frac{r}{a}\right)}{r}=\int d \widetilde{\omega} \Delta^{2} \frac{\varphi\left(t-\frac{r}{a}\right)}{r}+\Delta^{2} \int d \widetilde{\omega} \frac{\varphi(t)}{r}
$$

équation exacte pour des valeurs quelconques de $x, y, z$.

Si maintenant on multiplie $\left(3^{c}\right.$.) par la constante $a^{2}$, qu'on en déduise $\left(3^{a}\right.$.) et qu'on se serve de l'équation (3.) et du résultat connu:

$$
\triangle^{2} \int d \widetilde{\omega} \frac{\varphi(t, \alpha, \beta, \gamma)}{r}=-4 \pi \varphi(t, x, y, z) \text { ou }=0
$$

suivant que $(x, y, z)$ se trouve ou ne se trouve pas entre les limites de l'intégration, on parvient à l'équation

$$
\begin{gathered}
\text { (4.) } a^{2} \triangle^{2} \int d \widetilde{\omega} \frac{\varphi\left(t-\frac{r}{a}, \alpha, \beta, \gamma\right)}{r} \\
=\frac{d^{2}}{d t^{2}} \int d \widetilde{\varphi} \frac{\varphi\left(t-\frac{r}{a}, \alpha, \beta, \gamma\right)}{r}-4 \pi a^{2} \varphi(t, x, y, z),
\end{gathered}
$$

le point $(x, y, z)$ étant situé entre les limites de l'intégration.

Désignons par $\boldsymbol{X}_{2}(t), \boldsymbol{Y}_{2}(t), \boldsymbol{Z}_{2}(t)$ les fonctions qui satisfont aux équations

$$
\Delta^{2} \boldsymbol{X}_{2}(t)=\boldsymbol{X}(t), \quad \Delta^{2} \boldsymbol{Y}_{2}(t)=\boldsymbol{Y}(t), \quad \Delta^{2} \boldsymbol{Z}_{2}(t)=\boldsymbol{Z}(t),
$$

par $A_{2}(t), B_{2}(t), C_{2}(t)$ des fonctions correspondantes à celles-ci, $x, y, z$ 
étant remplacés par $\alpha, \beta, \gamma$, et posons, pour abréger,

$$
\begin{aligned}
& \mathrm{F}=\frac{1}{4 \pi \Omega^{2}} \int \frac{d \omega}{r}\left[\frac{d A_{2}\left(t-\frac{r}{\Omega}\right)}{d \alpha}+\frac{d B_{2}\left(t-\frac{r}{\Omega}\right)}{d \beta}+\frac{d C_{2}\left(t-\frac{r}{\Omega}\right)}{d \gamma}\right] \\
& L=\frac{1}{4 \pi \omega^{2}} \int \frac{d \omega}{r}\left[\frac{d B_{2}\left(t-\frac{r}{\omega}\right)}{d \gamma}-\frac{d C_{2}\left(t-\frac{r}{\omega}\right)}{d \beta}\right] \\
& M=\frac{1}{4 \pi \omega^{2}} \int \frac{d \omega}{r}\left[\frac{d C_{2}\left(t-\frac{r}{\omega}\right)}{d \alpha}-\frac{d A_{2}\left(t-\frac{r}{\omega}\right)}{d \gamma}\right] \\
& N=\frac{1}{4 \pi \omega^{2}} \int \frac{d \omega}{r}\left[\frac{d A_{2}\left(t-\frac{r}{\omega}\right)}{d \beta}-\frac{d B_{2}\left(t-\frac{r}{\omega}\right)}{d \alpha}\right]
\end{aligned}
$$

où

$$
\Omega^{2}=\frac{\lambda+2 \mu}{\rho}, \quad \omega^{2}=\frac{\mu}{\rho} .
$$

Les limites des intégrales sont les limites du corps élastique, et les dérivées partielles par rapport à $\alpha, \beta, \gamma$ sont prises, en conservant $r$ constant.

On trouvera donc que les valeurs

$$
\text { (5.) }\left\{\begin{array}{l}
u_{0}=\frac{d \mathrm{~F}}{d x}+\frac{d N}{d y}-\frac{d M}{d z} \\
v_{0}=\frac{d \mathrm{~F}}{d y}+\frac{d L}{d z}-\frac{d N}{d x} \\
w_{0}=\frac{d \mathrm{~F}}{d z}+\frac{d M}{d x}-\frac{d L}{d y}
\end{array}\right.
$$

satisfont aux équations (2.). La première équation (2.), par exemple, en y substituant ces valeurs de $u_{0}, v_{0}, w_{0}$, deviendra

$$
\Omega^{2} \triangle^{2} \frac{d \mathrm{~F}}{d x}+\omega^{2} \Delta^{2}\left[\frac{d N}{d y}-\frac{d M}{d z}\right]+X=\frac{d^{2}}{d t^{2}}\left[\frac{d \mathrm{~F}}{d x}+\frac{d N}{d y}-\frac{d M}{d z}\right]
$$

Mais, d'après l'équation (4.), on a

$$
\begin{aligned}
& \Omega^{2} \triangle^{2} F=\frac{d^{2} F}{d t^{2}}-\left(\frac{d X_{2}(t)}{d x}+\frac{d Y_{2}(t)}{d y}+\frac{d Z_{2}(t)}{d z}\right), \\
& \omega^{2} \triangle^{2} N=\frac{d^{2} N}{d t^{2}}-\frac{d X_{2}(t)}{d y}+\frac{d Y_{2}(t)}{d x}, \\
& \omega^{2} \triangle^{2} M=\frac{d^{2} M}{d t^{2}}-\frac{d Z_{2}(t)}{d x}+\frac{d X_{2}(t)}{d z},
\end{aligned}
$$

et notre équation se réduit donc à l'équation identique

$$
-\Delta^{2} \boldsymbol{X}_{2}(t)+\boldsymbol{X}=\mathbf{0} \text {. }
$$


Ainsi la première des équations (2.) est vérifiée et les deux autres peuvent l'être de la même manière.

Le problème de l'élimination des forces accélératrices étant donc complètement résolu, nous ferons dorénavant abstraction de ces forces.

Des mouvements d'un corps élastique illimité qui sont produits par les mouvements dans un plan.

Avant d'entrer dans la question dont il s'agit nous ferons quelques remarques mathématiques d'une grande importance dans la théorie de l'élasticité.

Une fonction finie de plusieurs variables $f\left(x_{1}, x_{2}, x_{3}, \ldots x_{n-1}\right)$ peut être exprimée par une intégrale définie de la manière suivante:

$$
\begin{gathered}
\text { (6.) } f\left(x_{1}, x_{2}, \ldots x_{n-1}\right) \\
=\frac{\Gamma\left(\frac{1}{2} n\right)}{\pi_{2}^{\frac{1}{2} n}}\left[\int d \alpha_{1} \int d \alpha_{2} \ldots \int d \alpha_{n-1} f\left(\alpha_{1}, \alpha_{2}, \ldots \alpha_{n-1}\right) \frac{x_{n}-\alpha_{n}}{r^{n}}\right]^{x_{n}=\alpha_{n}}
\end{gathered}
$$

où

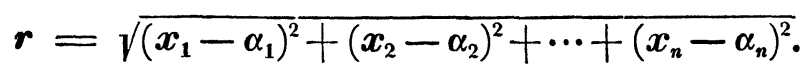

Dans cette formule les limites de l'intégration sont arbitraires et assujetties à la seule condition que les valeurs $\alpha_{1}=x_{1}, \alpha_{2}=x_{2}, \ldots \alpha_{n-1}=x_{n-1} y$ soient comprises. Cette condition sera done toujours remplie, si chacune des $n-1$ intégrations est faite entre les limites $-\infty$ et $+\infty$. De plus la différence $x_{n}-\alpha_{n}$ est supposée être positive avant d'être nulle.

On vérifie cette équation en considérant que, $x_{n}$ étant égal à $\alpha_{n}$, tous les éléments de l'intégrale s'évanouissent, excepté ceux pour lesquels $r$ est égal à zéro, d'où $x_{1}=\alpha_{1}, x_{2}=\alpha_{2}, \ldots x_{n-1}=\alpha_{n-1}$,

$$
f\left(\alpha_{1}, \alpha_{2}, \ldots \alpha_{n-1}\right)=f\left(x_{1}, x_{2}, \ldots x_{n-1}\right),
$$

et en se servant du résultat connu d'après lequel l'intégrale

$$
\int_{-\infty}^{+\infty} d \alpha_{1} \int_{-\infty}^{+\infty} d \alpha_{2} \ldots \int_{-\infty}^{+\infty} d \alpha_{n-1} \frac{x_{n}-\alpha_{n}}{r^{n}}
$$

qui se transforme en

$$
\int_{-\infty}^{+\infty} d \xi_{1} \int_{-\infty}^{+\infty} d \xi_{2} \ldots \int_{-\infty}^{+\infty} d \xi_{n-1} \frac{1}{\left\{1+\xi_{1}^{2}+\xi_{2}^{2}+\cdots+\xi_{n-1}^{2}\right\}^{\frac{1}{2} n}}
$$

par les substitutions $\alpha_{1}-x_{1}=\xi_{1}\left(x_{n}-\alpha_{n}\right), \ldots \alpha_{n-1}-x_{n-1}=\xi_{n-1}\left(x_{n}-\alpha_{n}\right)$ est égale à $\frac{\pi^{\text {tn }}}{\Gamma\left(\frac{1}{2} n\right)}(J a c o b i$ de transform. integr. mult. , vol. 12, pag. 60 de ce Journal, où l'intégrale dont il s'agit est désignée par $2^{n-1} \boldsymbol{S}$ ). 
Il est bon d'observer que l'expression comprise entre les crochets (6.) satisfait à l'équation aux différences partielles

$$
\frac{d^{2}}{d x_{1}^{2}}+\frac{d^{2}}{d x_{2}^{2}}+\cdots+\frac{d^{2}}{d x_{n}^{2}}=0
$$

Une fonction de deux variables $f(x, y)$ sera donc exprimée par $f(x, y)=\frac{1}{2 \pi}\left[\int d \alpha \int d \beta \frac{z-\gamma}{r^{3}} f(\alpha, \beta)\right]^{z=\gamma}, r=\sqrt{(x-\alpha)^{2}+(y-\beta)^{2}+(z-\gamma)^{2}}$ ou

$$
\text { (7.) } f(x, y)=-\frac{1}{2 \pi}\left[\frac{d}{d z} \int d \alpha \int d \beta \frac{f(\alpha, \beta)}{r}\right]^{z=\gamma}
$$

le point $(x, y)$ étant situé entre les limites de l'intégration relative à $\alpha, \beta$, et $z-\gamma$ étant supposé positif avant d'être nul.

L'expression comprise entre les crochets satisfait à l'équation différentielle $\Delta^{2}=0$, et elle exprime l'attraction que d'après la loi Newtonienne un plan exerce sur un point dans la direction de la normale du plan, la masse d'un élément étant $f(\alpha, \beta) d \alpha d \beta$, et l'attraction exercée par l'unité de masse dans l'unité de distance étant égale à l'unité. La formule (7.) équivaut donc à l'énoncé, que l'attraction que le plan exerce sur un point infiniment rapproché, dans la direction de la normale, est égale à $2 \pi f(x, y)$, c'est-à-dire, à la masse de l'élément-plan $d x d y$ divisée par $\frac{1}{2 \pi} d x d y$. Supposons qu'il s'agisse de satisfaire à l'équation différentielle

$$
a^{2} \Delta^{2} \mathrm{~F}=\frac{d^{2} \mathrm{~F}}{d t^{2}}
$$

$a$ étant une constante et que la fonction $F$ soit en outre déterminée par la condition que pour un plan arbitrairement limité dans lequel nous plaçons le, plan coordonné $(y, z)$ et pour des valeurs quelconques du temps $t$ elle se réduise à une fonction donnée de $y, z$ et $t$, de sorte que l'on ait:

$$
[\mathrm{F}]^{x=0}=\boldsymbol{F}(t, y, z) \text {, }
$$

cela posé le mouvement sera déterminé dans tout l'espace du côté positif du plan coordonné $(y, z)$ par l'équation

$$
\text { (8.) }\left\{\begin{array}{l}
\mathrm{F}=-\frac{1}{2 \pi} \frac{d}{d x} \int d \alpha \int d \beta \frac{F\left(t-\frac{r}{a}, \beta, \gamma\right)}{r}, \\
r=\sqrt{x^{2}+(y-\beta)^{2}+(z-\gamma)^{2}}
\end{array}\right.
$$

les limites de l'intégrale étant celles du plan donné. Cette expression satis- 
fera à l'équation différentielle et, pour $x=0$, on aura

$$
\begin{aligned}
{[\mathrm{F}]^{x=0} } & =-\frac{1}{2 \pi}\left[\frac{d}{d x} \int d \alpha \int d \beta \frac{F\left(t-\frac{r}{a}, \beta, \gamma\right)}{r}\right]^{x=0} \\
& =-\frac{1}{2 \pi}\left[\frac{d}{d x} \int d \alpha \int d \beta \frac{F(t, \beta, \gamma)}{r}\right]^{x=0},
\end{aligned}
$$

d'où, après la formule (7.),

$$
[\mathrm{F}]^{x=0}=\boldsymbol{F}(t, y, z),
$$

ce qui est la condition donnée.

Nous sommes donc en état de résoudre, par la méthode indiquée, un problème, que l'on n'a résolu jusqu'ici que d'une manière inexacte et incomplète moyennant le principe de Huyghens.

L'intégrale $F$ nous fait voir que le mouvement peut être considéré comme partant de chaque élément-plan $d \beta d \gamma$ avec une vitesse constante, égale à $a$.

Passons maintenant aux trois équations (2.) du mouvement, en faisant abstraction des forces accélératrices. En les ajoutant, après les avoir respectivement différentiées par rapport à $x, y$ et $z$, on obtient, comme on sait,

$$
\frac{\lambda+2 \mu}{\varrho} \Delta^{2} \theta=\frac{d^{2} \theta}{d t^{2}}
$$

par conséquent $\sqrt{ } \frac{\lambda+2 \mu}{\rho}=\Omega$ est la vitesse avec laquelle chaque condensation ou dilatation se propage dans le corps élastique.

Par l'élimination de $\theta$ on déduit des équations (2.) le résultat

$$
\frac{\mu}{\varrho} \Delta^{2} \varphi=\frac{d^{2} \varphi}{d t^{2}}, \quad \varphi=\left\{\begin{array}{l}
\frac{d v}{d z}-\frac{d w}{d y} \\
\frac{d w}{d x}-\frac{d u}{d z} \\
\frac{d u}{d y}-\frac{d v}{d x}
\end{array}\right.
$$

Il y a donc une autre espèce de mouvement, qui se propage avec la vitesse $\sqrt{\frac{\mu}{\rho}}=\omega$.

Pour la première espèce de mouvement, il faut avoir $\varphi=0$, et par là

(9.) $u=\frac{d \mathrm{~F}}{d x}, \quad v=\frac{d \mathrm{~F}}{d y}, \quad w=\frac{d \mathrm{~F}}{d z}, \quad \Omega^{2} \Delta^{2} \mathrm{~F}=\frac{d^{2} \mathrm{~F}}{d t^{2}}$.

L'autre fera 


$$
\left\{\begin{array}{l}
\theta=\frac{d u}{d x}+\frac{d v}{d y}+\frac{d w}{d z}=0, \\
\mathrm{~d}^{\prime} \mathrm{où} \\
\omega^{2} \triangle^{2} u=\frac{d^{2} u}{d t^{2}}, \quad \omega^{2} \triangle^{2} v=\frac{d^{2} v}{d t^{2}}, \quad \omega^{2} \triangle^{2} w=\frac{d^{2} w}{d t^{2}} .
\end{array}\right.
$$

Désignons maintenant par $u, v, w$ les projections des déplacements, qui dépendent de $\omega$; par $u^{\prime}, v^{\prime}, w^{\prime}$ celles qui dépendent de $\Omega$; par $U, V, W$ la somme de ces deux, et introduisons les nouvelles fonctions:

$$
\begin{gathered}
\Phi=-\frac{1}{2 \pi} \int d \beta \int d \gamma \frac{\varphi\left(t-\frac{r}{\omega}, \beta, \gamma\right)}{r}, \quad \Phi^{\prime}=-\frac{1}{2 \pi} \int d \beta \int d \gamma \frac{\varphi\left(t-\frac{r}{\Omega}, \beta, \gamma\right)}{r}, \\
\Psi=-\frac{1}{2 \pi} \int d \beta \int d \gamma \frac{\psi\left(t-\frac{r}{\omega}, \beta, \gamma\right)}{r}, \quad \Psi^{\prime}=-\frac{1}{2 \pi} \int d \beta \int d \gamma \frac{\psi\left(t-\frac{r}{\Omega}, \beta, \gamma\right)}{r}, \\
X=-\frac{1}{2 \pi} \int d \beta \int d \gamma \frac{\chi\left(t-\frac{r}{\omega}, \beta, \gamma\right)}{r}, \quad X^{\prime}=-\frac{1}{2 \pi} \int d \beta \int d \gamma \frac{\chi\left(t-\frac{r}{\Omega}, \beta, \gamma\right)}{r}, \\
r=\sqrt{x^{2}+(y-\beta)^{2}+(z-\gamma)^{2}} .
\end{gathered}
$$

Nous verrons, que l'on pourra déterminer les mouvements d'un corps élastique illimité, si l'on connait, dans le plan coordonné $(y, z)$, ou la pression normale et les déplacements tangentiels, ou les pressions tangentielles et le déplacement normal. Dans le premier cas nous posons

d'où

$$
\begin{cases}u=\Phi-\frac{d \mathrm{~F}}{d x}, & u^{\prime}=\frac{d \mathrm{~F}^{\prime}}{d x} \\ v=\frac{d \Psi}{d x}-\frac{d \mathrm{~F}}{d y}, & v^{\prime}=\frac{d \mathrm{~F}^{\prime}}{d y} \\ w=\frac{d \mathrm{X}}{d x}-\frac{d \mathrm{~F}}{d z}, & \boldsymbol{w}^{\prime}=\frac{d \mathrm{~F}^{\prime}}{d z}\end{cases}
$$

$$
\begin{aligned}
& \triangle^{2} \mathrm{~F}=\frac{d}{d x}\left[\Phi+\frac{d \Psi}{d y}+\frac{d X}{d z}\right]=\frac{1}{\omega^{2}} \frac{d^{2} \mathrm{~F}}{d t^{2}}, \\
& \triangle^{2} \mathrm{~F}^{\prime}=\frac{\omega^{2}}{\Omega^{2}} \frac{d}{d x}\left[\Phi^{\prime}+\frac{d \Psi}{d y}+\frac{d X^{\prime}}{d z}\right]=\frac{1}{\Omega^{2}} \frac{d^{2} F^{\prime}}{d t^{2}} .
\end{aligned}
$$

Ces valeurs satisfont aux équations générales du mouvement (9.) et (10.), et, pour $x$ égal à zéro, on obtient

$$
\begin{aligned}
{\left[\frac{d u}{d x}+\frac{d u^{\prime}}{d x}\right]^{x=0} } & =\left[\frac{d U}{d x}\right]^{x=0}=\frac{\omega^{2}}{\Omega^{2}} \varphi(t, y, z)-\frac{\Omega^{2}-\omega^{2}}{\Omega^{2}}\left[\frac{d \psi(t, y, z)}{d y}+\frac{d \chi(t, y, z)}{d z}\right], \\
{\left[v+v^{\prime}\right]^{x=1} } & =[\boldsymbol{V}]^{x=0}=\psi(t, y, z), \\
{\left[w+w^{\prime}\right]^{x=0} } & =[W]^{x=0}=\chi(t, y, z) .
\end{aligned}
$$


Les fonctions $\varphi, \psi$ et $\chi$ seront donc déterminées par les fonctions $\left[\frac{d U}{d x}\right]^{x=0}[V]^{x=0}$ et $[W]^{x=0}$. Si l'on connaît, au lieu de $\left[\frac{d U}{d x}\right]^{x=0}$, la pression normale $\left[\boldsymbol{N}_{1}\right]^{x=0}$, on aura

$$
\left[N_{1}\right]^{x=0}=\left[\lambda \theta+2 \mu \frac{d U}{d x}\right]^{x=0}=\mu\left[\varphi(t, y, z)-\frac{d \psi(t, y, z)}{d y}-\frac{d \chi(t, y, z)}{d z}\right],
$$

équation par laquelle la fonction $\varphi$ est déterminée.

Cependant, nous n'avons pas fait attention à la fonction arbitraire qui doit entrer dans les valeurs des composantes. Ces valeurs arbitraires de $u, v, w$, qu'il faut ajouter aux valeurs trouvées, seront

$$
\begin{array}{r}
u=\frac{1}{2 \pi} \frac{d}{d y} \int d \beta \int d \gamma \frac{1}{r} \frac{d f\left(t-\frac{r}{\omega}, \beta, \gamma\right)}{d \gamma} \\
-\frac{1}{2 \pi} \frac{d}{d z} \int d \beta \int d \gamma \frac{1}{r} \frac{d f\left(t-\frac{r}{\omega}, \beta, \gamma\right)}{d \beta}, \\
v=\frac{1}{2 \pi} \frac{d^{2}}{d x d z} \int d \beta \int d \gamma \frac{f\left(t-\frac{r}{\omega}, \beta, \gamma\right)}{r} \\
\quad-\frac{1}{2 \pi} \frac{d}{d x} \int d \beta \int d \gamma \frac{1}{r} \frac{d f\left(t-\frac{r}{\omega}, \beta, \gamma\right)}{d \gamma}, \\
w=-\frac{1}{2 \pi} \frac{d^{2}}{d x d y} \int d \beta \int d \gamma \frac{t\left(t-\frac{r}{\omega}, \beta, \gamma\right)}{r} \\
\quad+\frac{1}{2 \pi} \frac{d}{d x} \int d \beta \int d \gamma \frac{1}{r} \frac{d f\left(t-\frac{r}{\omega}, \beta, \gamma\right)}{d \beta},
\end{array}
$$

où les dérivées partielles par rapport à $\beta$ et $\gamma$ sont prises en conservant $r$ constant. On voit que ces valeurs feront

$$
\theta=0, \quad\left[\frac{d u}{d x}\right]^{x=0}=0, \quad[v]^{x=0}=0, \quad[w]^{x=0}=0 .
$$

En intégrant par partie et désignant les limites de $\beta$ et $\gamma$ par $\beta_{0}, \beta_{1}$ et $\gamma_{0}, \gamma_{1}$, on pourra mettre les expressions sous cette autre forme 


$$
\left\{\begin{array}{r}
u=\frac{1}{2 \pi} \frac{d}{d y} \int d \beta\left[\frac{f\left(t-\frac{r}{\omega}, \beta, \gamma\right)}{r}\right]_{\gamma=\gamma_{0}}^{\gamma=\gamma_{1}}, \\
-\frac{1}{2 \pi} \frac{d}{d z} \int d \gamma\left[\frac{f\left(t-\frac{r}{\omega}, \beta, \gamma\right)}{r}\right]_{\beta=\beta_{1}}^{\beta=\beta_{0}}, \\
v=-\frac{1}{2 \pi} \frac{d}{d x} \int d \beta\left[\frac{f\left(t-\frac{r}{\omega}, \beta, \gamma\right)}{r}\right]_{\gamma=\gamma_{1}}^{\gamma=\gamma_{1}}, \\
w=-\frac{1}{2 \pi} \frac{d}{d x} \int d \gamma\left[\frac{f\left(t-\frac{r}{\omega}, \beta, \gamma\right)}{r}\right]_{\beta=\beta_{1}}^{\beta=\beta_{0}},
\end{array}\right.
$$

Dans le deuxième cas, si l'on connaît dans le plan coordonné $(y, z)$ les pressions tangentielles et le déplacement normal; il faut poser

$$
\text { (13.) }\left\{\begin{aligned}
u=\frac{d \Phi}{d x}-\frac{d \mathrm{~F}}{d x}, & u^{\prime}=\frac{d \mathrm{~F}^{\prime}}{d x}, \\
v=\Psi-\frac{d \mathrm{~F}}{d y}, & v^{\prime}=\frac{d \mathrm{~F}^{\prime}}{d y}, \\
w=X-\frac{d \mathrm{~F}}{d z}, & \boldsymbol{w}^{\prime}=\frac{d \mathrm{~F}^{\prime}}{d z},
\end{aligned}\right.
$$

d'où

$$
\begin{aligned}
& \triangle^{2} \mathrm{~F}=\frac{d^{2} \Psi}{d x^{2}}+\frac{d \Psi}{d y}+\frac{d X}{d z}=\frac{1}{\omega^{2}} \frac{d^{2} \mathrm{~F}}{d t^{2}} \\
& \triangle^{2} \mathrm{~F}^{\prime}=\frac{\omega^{2}}{\Omega^{2}}\left[\frac{d^{2} \Phi^{\prime}}{d x^{2}}+\frac{d \Psi \prime}{d y}+\frac{d X^{\prime}}{d z}\right]=\frac{1}{\Omega^{2}} \frac{d^{2} \mathrm{~F}^{\prime}}{d t^{2}} .
\end{aligned}
$$

Les fonctions $\varphi, \psi$ et $\chi$ seront donc déterminées par

$$
\begin{aligned}
& \varphi(t, y, z)=\frac{\Omega^{2}}{\omega^{2}}[U]^{x=0}, \\
& \psi(t, y, z)=\left[\frac{d V}{d x}\right]^{x=0}+\frac{\Omega^{2}-\omega^{2}}{\omega^{2}} \frac{d}{d y}[U]^{x=0}, \\
& \chi(t, y, z)=\left[\frac{d W}{d x}\right]^{x=0}+\frac{\Omega^{2}-\omega^{2}}{\omega^{2}} \frac{d}{d z}[U]^{x=0} .
\end{aligned}
$$

Or, les pressions tangentielles

$$
\left[T_{3}\right]^{x=0}=\mu\left[\frac{d U}{d y}+\frac{d V}{d x}\right]^{x=0}, \quad\left[T_{2}\right]^{x=0}=\mu\left[\frac{d U}{d z}+\frac{d W}{d x}\right]^{x=0}
$$

étant connues, on trouvera 


$$
\begin{aligned}
& \varphi(t, y, z)=\frac{\lambda+2 \mu}{\mu}[\boldsymbol{U}]^{x=0}, \\
& \psi(t, y, z)=\frac{1}{\mu}\left[T_{3}\right]^{x=0}+\frac{\lambda}{\mu} \frac{d}{d y}[\boldsymbol{U}]^{x=0}, \\
& \chi(t, y, z)=\frac{1}{\mu}\left[T_{2}\right]^{x=0}+\frac{\lambda}{\mu} \frac{d}{d z}[U]^{x=0} .
\end{aligned}
$$

Les valeurs arbitraires des composantes qu'il faut ajouter à ces valeurs, seront les dérivées par rapport à $x$ des valeurs arbitraires trouvées ci-dessus (12.).

\section{3.}

\section{Diff raction.}

Le mouvement vibratoire d'un corps élastique et illimité, ayant traversé une ouverture d'un plan fixe, serait déterminé par les méthodes indiquées, si l'on pouvait considérer comme connu le mouvement qui s'opère dans l'ouverture. Mais cela n'est vrai que d'une manière approximative, car le mouvement dans l'ouverture n'est pas exactement le même, que celui qui aurait lieu, s'il n'y avait pas de plan fixe. On aura un mouvement réfléchi de l'ouverture en même temps qu'un mouvement transmis, et, pour déterminer tous les deux, on aura les conditions suivantes: $1^{\circ}$ la somme des composantes du mouvement direct et réfléchi est égale aux composantes du mouvement transmis à travers l'ouverture, et $2^{\circ}$ les pressions normales et tangentielles sur les deux faces du plan qui coïncide avec l'ouverture sont égales dans chaque point.

Désignons par $\boldsymbol{U}, \boldsymbol{V}, \boldsymbol{W}$ les composantes du mouvement direct, par $U_{1}, V_{1}, W_{1}$ celles du mouvement transmis, par $U_{2}, V_{2}, W_{2}$ celles du mouvement réfléchi, et par $\theta, \theta_{1}, \theta_{2}$ les dilatations correspondantes à ces trois mouvements. Faisons coïncider le plan coordonné $(y, z)$ avec l'ouverture. Cela posé nous aurons pour $x=0$

$$
\text { (14.) }\left[U+U_{2}-U_{1}\right]^{x=0}=0,\left[V+V_{2}-V_{1}\right]^{x=0}=0,\left[W+W_{2}-W_{1}\right]^{x=0}=0
$$

et de plus

$$
\begin{aligned}
& {\left[\lambda\left(\theta+\theta_{2}-\theta_{1}\right)+2 \mu \frac{d\left(U+U_{2}-U_{1}\right)}{d x}\right]^{x=0}=0,} \\
& {\left[\frac{d\left(U+U_{2}-U_{1}\right)}{d y}+\frac{d\left(V+V_{2}-V_{1}\right)}{d x}\right]^{x=0}=0,} \\
& {\left[\frac{d\left(U+U_{2}-U_{1}\right)}{d z}+\frac{d\left(W+W_{2}-W_{1}\right)}{d x}\right]^{x=0}=0 .}
\end{aligned}
$$

Les trois dernières équations peuvent être simplifiées, au moyen des trois 
premières, dérivées par rapport à $y$ ou $z$; de cette manière elles se réduisent aux équations suivantes:

$$
\left\{\begin{array}{l}
{\left[\frac{d\left(U+U_{2}-U_{1}\right)}{d x}\right]^{x=0}=0} \\
{\left[\frac{d\left(V+V_{2}-V_{1}\right)}{d x}\right]^{x=0}=0} \\
{\left[\frac{d\left(W+W_{2}-W_{1}\right)}{d x}\right]^{x=0}=0}
\end{array}\right.
$$

Introduisons les notations du numéro précédent et posons

$$
\begin{array}{lll}
U_{1}=u_{1}+u_{1}^{\prime}, & V_{1}=v_{1}+v_{1}^{\prime}, & W_{1}=w_{1}+w_{1}^{\prime}, \\
U_{2}=u_{2}+u_{2}^{\prime}, & V_{2}=v_{2}+v_{2}^{\prime}, & W_{2}=w_{2}+w_{2}^{\prime}, \\
u_{1}^{\prime}=\frac{d \mathrm{~F}_{1}^{\prime}}{d x}, & v_{1}^{\prime}=\frac{d \mathrm{~F}_{1}^{\prime}}{d y}, & \boldsymbol{u}_{1}^{\prime}=\frac{d \mathrm{~F}_{1}^{\prime}}{d z}, \\
u_{2}^{\prime}=\frac{d \mathrm{~F}_{2}^{\prime}}{d x}, & v_{2}^{\prime}=\frac{d \mathrm{~F}_{2}^{\prime}}{d y}, & \boldsymbol{w}_{2}^{\prime}=\frac{d \mathrm{~F}_{2}^{\prime}}{d z} .
\end{array}
$$

Le mouvement direct peut être supposé dépendant ou de la vitesse $\Omega$, ou de la vitesse $\omega$. Dans le premier cas nous posons

$$
\mathrm{U}=\frac{d \mathrm{~F}^{\prime}}{d x}, \quad V=\frac{d \mathrm{~F}^{\prime}}{d y}, \quad W=\frac{d \mathrm{~F}^{\prime}}{d z} .
$$

On trouvera, que les composantes $u_{1}, u_{2}$, etc., qui dépendent de $\omega$, s'évanouissent, et les équátions (14.) et (15.) deviendront

$$
\left[\mathrm{F}^{\prime}+\mathrm{F}_{2}^{\prime}-\mathrm{F}_{1}^{\prime}\right]^{x=10}=0, \quad\left[\frac{d\left(\mathrm{~F}^{\prime}+\mathrm{F}_{2}^{\prime}-\mathrm{F}_{1}^{\prime}\right)}{d x}\right]^{x=0}=0 .
$$

Notons les équations

$$
\left[\frac{d \Psi^{\prime}}{d x}\right]^{x=0}=-\psi(t, y, z), \quad\left[\frac{d \Phi^{\prime}}{d x}\right]^{x=0}=-\varphi(t, y, z),
$$

dans lesquelles $x$ est supposé s'évanouir, après avoir eu une valeur négative, et posons

$$
\text { (16.) } \quad F_{2}^{\prime}=\Phi^{\prime}+\frac{d \Psi \prime}{d x}, \quad F_{1}^{\prime}=\Phi^{\prime}+\frac{d \Psi '}{d x} \text {. }
$$

Ces valeurs étant identiques pour les deux fonctions $F_{2}^{\prime}$ et $F_{1}^{\prime}$ on a

$$
\begin{aligned}
& {\left[\mathrm{F}_{1}^{\prime}-\mathrm{F}_{2}^{\prime}\right]^{x=0} }=\left[\mathrm{F}^{\prime}\right]^{x=0}=2 \psi(t, y, z), \\
& {\left[\frac{d\left(\mathrm{~F}_{1}^{\prime}-\mathrm{F}_{2}^{\prime}\right)}{d x}\right]^{x=0}=\left[\frac{d \mathrm{~F}^{\prime}}{d x}\right]^{x=0}=2 \varphi(t, y, z) . }
\end{aligned}
$$

Les équations précédentes déterminent les fonctions $\psi$ et $\varphi$, et par conséquent les fonctions $\Phi^{\prime}$ et $\Psi^{\prime}$. 
Dans l'autre cas, le mouvement direct étant dépendant de la vitesse $\omega$, nous avons

$$
U=u, \quad V=v, \quad W=w, \quad \frac{d u}{d x}+\frac{d v}{d y}+\frac{d w}{d z}=0 .
$$

Les fonctions $F_{1}^{\prime}$ et $F_{2}^{\prime}$ s'évanouissent, et les équations (14.) et (15.) deviennent

$$
\begin{gathered}
{\left[u+u_{2}-u_{1}\right]^{x=0}=0, \quad\left[v+v_{2}-v_{1}\right]^{x=0}=0, \quad\left[w+w_{2}-w_{1}\right]^{x=0}=0,} \\
{\left[\frac{d\left(u+u_{2}-u_{1}\right)}{d x}\right]^{x=0}=0, \quad\left[\frac{d\left(v+v_{2}-v_{1}\right)}{d x}\right]^{x=0}=0, \quad\left[\frac{d\left(w+w_{2}-w_{1}\right)}{d x}\right]^{x=0}=0 .}
\end{gathered}
$$

Introduisons les fonctions $\Phi_{1}, \Psi_{1}, X_{1}$, en les faisant dépendre des nouvelles fonctions $\varphi_{1}, \psi_{1}, \chi_{1}$, de la manière que, dans le numéro précédent, on a fait dépendre $\Phi, \Psi, X$ de $\varphi, \psi, \chi$, et posons

d'où

$$
\begin{aligned}
& u_{1}=\Phi+\frac{d \Phi_{1}}{d x}-\frac{d\left(\mathrm{~F}+\mathrm{F}_{1}\right)}{d x} \\
& v_{1}=\Psi+\frac{d \Psi_{1}}{d x}-\frac{d\left(\mathrm{~F}+\mathrm{F}_{1}\right)}{d y} \\
& w_{1}=X+\frac{d X_{1}}{d x}-\frac{d\left(\mathrm{~F}+\mathrm{F}_{1}\right)}{d z}
\end{aligned}
$$

$$
\begin{aligned}
& \triangle^{2} \mathrm{~F}=\frac{d}{d x}\left[\Phi+\frac{d \Psi_{1}}{d y}+\frac{d \mathrm{X}_{1}}{d z}\right]=\frac{1}{\omega^{2}} \frac{d^{2} \mathrm{~F}}{d t^{2}} \\
& \triangle^{2} \mathrm{~F}_{1}=\frac{d^{2} \Phi_{1}}{d x^{2}}+\frac{d \Psi}{d y}+\frac{d X}{d z}=\frac{1}{\omega^{2}} \frac{d^{2} \mathrm{~F}_{1}}{d t^{2}} .
\end{aligned}
$$

Aux composantes du mouvement réfléchi c. à. d. aux quantités $u_{2}, v_{2}, w_{2}$ nous attribuons les mêmes valeurs, avec la seule différence de prendre $x$ toujours négatif.

En supposant, que

nous trouverons

$$
\text { (18.) }[\mathrm{F}]^{x=0}=0,\left[\frac{d \mathrm{~F}_{1}}{d x}\right]^{x=0}=0,
$$

$$
\begin{array}{r}
{\left[u+u_{2}-u_{1}\right]^{x=0}=[u]^{x=0}-2 \varphi_{1}(t, y, z)=0,} \\
{\left[v+v_{2}-v_{1}\right]^{x=0}=[v]^{x=0}-2 \psi_{1}(t, y, z)=0,} \\
{\left[w+w_{2}-w_{1}\right]^{x=0}=[\boldsymbol{w}]^{x=0}-2 \chi_{1}(t, y, z)=0,} \\
{\left[\frac{d\left(u+u_{2}-u_{1}\right)}{d x}\right]^{x=0}=\left[\frac{d u}{d x}\right]^{x=0}-2 \varphi(t, y, z)=0,} \\
{\left[\frac{d\left(v+v_{2}-v_{1}\right)}{d x}\right]^{x=0}=\left[\frac{d v}{d x}\right]^{x=0}-2 \psi(t, y, z)=0,} \\
{\left[\frac{d\left(w+w_{2}-w_{1}\right)}{d x}\right]^{x=0}=\left[\frac{d w}{d x}\right]^{x=0}-2 \chi(t, y, z)=0 .}
\end{array}
$$


Les fonctions $\varphi, \varphi_{1}$, etc. seront donc déterminées, et maintenant on vérifiera aisément les équations (18.). Si par exemple le mouvement direct forme une onde plane, on trouvera pour l'onde transmise à des distances assez grandes de l'ouverture les mêmes expressions, qu'a déjà trouvées M. Stokes d'une manière moins rigoureuse.

\section{4. \\ Tuyaux sonores.}

Considérons maintenant les petits mouvements vibratoires dans un corps élastique et complètement fluide entre des plans fixes qui permettent aux points contigus de faire librement tous les mouvements parallèles au plan en excluant le mouvement normal. Quand le corps élastique est complétement fluide, la constante $\mu$ est égale à zéro, et il n'y existe qu'une espèce de vibration, c'est-à-dire celle qui dépend de la vitesse $\Omega$.

En désignant par $u, v, w$ les composantes des déplacements, nous posons

$$
\frac{d \mathrm{~F}}{d x}=u, \quad \frac{d \mathrm{~F}}{d y}=v, \quad \frac{d \mathrm{~F}}{d z}=w,
$$

et donnons à la fonction $\mathrm{F}$ le nom de potentiel de l'onde ou du mouvement.

Supposons que le corps fluide soit renfermé dans un tuyau d'une longueur illimitée et à base rectangle. Faisons coïncider ses parois avec les plans coordonnés $(y=0)$ et $(z=0)$ et avec les plans parallèles à ces derniers $(y=b)$ et $(z=c)$. Le potentiel du mouvement direct et de tous les mouvements réfléchis pourra donc être de la forme

$$
\left\{\begin{array}{l}
\mathrm{F}=-\frac{1}{2 \pi} \Sigma \Sigma \int_{0}^{b} d \beta \int_{0}^{c} d \gamma \frac{f\left(t-\frac{r}{\Omega}, \beta, \gamma\right)}{r} \\
r=\sqrt{x^{2}+\left(y+2 i_{1} b \pm \beta\right)^{2}+\left(z+2 i_{2} c \pm \gamma\right)^{2}}
\end{array}\right.
$$

$\Sigma \Sigma$ désignant la somme pour toutes les valeurs entières, négatives et positives, de $i_{1}$ et $i_{2}$, et le signe \pm , introduit par $r$ dans la fonction $F$, ayant ici, comme dans ce qui suit une signification spéciale, définie par

$$
\text { fonct. }( \pm)=\text { fonct. }(t)+\text { fonct. }(-) \text {. }
$$

Cette valeur de $F$ satisfait à l'équation différentielle

et donne

$$
\Delta^{2} \mathrm{~F}=\frac{1}{\Omega^{2}} \frac{d^{2} \mathrm{~F}}{d t^{2}}
$$

$$
[u]^{x=0}=f(t, y, z), \quad[v]^{p=0}=0, \quad[v]^{y=b}=0, \quad[w]^{z=0}=0, \quad[\boldsymbol{w}]^{z=c}=0 .
$$


Supposons à présent que le tuyau soit limité, qu'il soit coupé normalement à l'axe et forme un prisme droit de la longueur a. Faisons coïncider avec le plan coordonné $(x=0)$ son premier bout qui pourra être fermé ou ouvert, mais supposons que l'autre bout soit ouvert. Cela posé, le mouvement produit au premier bout se propagera à l'autre bout ouvert, et là il sera en partie réfléchi, en partie transmis au corps fluide illimité qui se trouve hors du tuyau.

Les composantes des vibrations réfléchies étant

$$
u_{2}=\frac{d \mathrm{~F}_{2}}{d x}, \quad v_{2}=\frac{d \mathrm{~F}_{2}}{d y}, \quad w_{2}=\frac{d \mathrm{~F}_{2}}{d z}
$$

et celles des vibrations transmises

$$
u_{1}=\frac{d \mathrm{~F}_{1}}{d x}, \quad v_{1}=\frac{d \mathrm{~F}_{1}}{d y}, \quad w_{1}=\frac{d \mathrm{~F}_{1}}{d z},
$$

nous aurons, comme dans le numéro précédent, les conditions suivantes:

$$
\begin{aligned}
& \text { (20.) }\left[\mathrm{F}+\mathrm{F}_{2}-\mathrm{F}_{1}\right]^{x=a}=0, \\
& \text { (21.) }\left[\frac{d\left(\mathrm{~F}+\mathrm{F}_{2}-\mathrm{F}_{1}\right)}{d x}\right]^{x=a}=0 .
\end{aligned}
$$

Posons maintenant

et

$$
\left\{\begin{array}{l}
\mathbf{F}_{2}=\frac{1}{2 \pi} \Sigma \Sigma \int_{0}^{b} d \beta \int_{0}^{c} d \gamma \frac{f_{2}\left(t-\frac{r}{\Omega}, \beta, \gamma\right)}{r} \\
r=\sqrt{(x-a)^{2}+\left(y+2 i_{1} b \pm \beta\right)^{2}+\left(z+2 i_{2} c \pm \gamma\right)^{2}}
\end{array}\right.
$$

(23.) $\left\{\begin{array}{l}\mathrm{F}_{1}=-\frac{1}{2 \pi} \int_{0}^{b} d \beta \int_{0}^{c} d \gamma \frac{f_{1}\left(t-\frac{r}{\Omega}, \beta, \gamma\right)}{r}, \\ r=\sqrt{(x-a)^{2}+(x-\beta)^{2}+(z-\gamma)^{2}} .\end{array}\right.$

Ces valeurs de $F_{2}$ et $F_{1}$, introduites dans (21.), nous donnent l'équation

$$
\left[\frac{d \mathrm{~F}}{d x}\right]^{x=a}+f_{2}(t, y, z)-f_{1}(t, y, z)=0
$$

par laquelle on peut éliminer l'une des fonctions $f_{1}$ ou $f_{2}$; l'autre doit donc être déterminée par l'équation (20.).

Mais, en général, on ne peut pas effectuer cette détermination. Nous chercherons donc une solution approximative, en supposant, que tous les déplacements entre les parois du tuyau se fassent seulement dans la direction de l'axe du tuyau. Cette hypothèse, qui ne s'accorde pas exactement avec l'équation (20.), nous conduira au résultat le plus juste, si nous égalons à zéro 
la somme des erreurs pour toutes les valeurs des $y$ et $z$. L'équation (20.) doit donc être remplaçée par cette autre

$$
\text { (25.) } \int_{0}^{b} d y \int_{0}^{c} d z\left[F+F_{2}-F_{1}\right]^{x=a}=0 \text {. }
$$

Maintenant le calcul peut s'étendre à des tuyaux d'une base quelconque, et nous pouvons poser

$$
\begin{aligned}
& \mathrm{F}=h \cos k(\Omega t-x), \\
& \mathrm{F}_{2}=h_{2} \cos k(\Omega t+x-2 a-\Delta), \\
& \left\{\begin{array}{l}
\mathrm{F}_{1}=-\frac{1}{2 \pi} h_{1} k \int d \beta \int d \gamma \frac{\sin k(\Omega t-r-a-\delta)}{r}, \\
r=\sqrt{(x-a)^{2}+(y-\beta)^{2}+(z-\gamma)^{2}},
\end{array}\right.
\end{aligned}
$$

en déterminant les constantes $h_{2}, h_{1}, \mathcal{A}, \delta$ par les équations

et

$$
\text { (21.) }\left[\frac{d\left(\mathrm{~F}+\mathrm{F}_{2}-\mathrm{F}_{1}\right)}{d x}\right]^{x=a}=0
$$

$$
\text { (29.) } \int d y \int d z\left[F+F_{2}-F_{1}\right]^{x=a}=0 \text {, }
$$

les intégrations devant être étendues jusqu'aux limites de la base.

En dévéloppant $\sin k(\Omega \ell-r-a-\delta)$ suivant les puissances ascendantes de $r$, on trouvera

$$
\int d y \int d z\left[\mathrm{~F}_{1}\right]^{x=a}=h_{1} B\left[-\varepsilon \sin k(\Omega t-a-\delta)+\varepsilon^{\prime} \cos k(\Omega t-a-\delta)\right],
$$

$B$ étant l'aire de la base, $\varepsilon$ et $\varepsilon^{\prime}$ des constantes positives. Nous aurons donc, en vertu des équations (21.) et (29.)

$$
h \sin k(\Omega t-a)-h_{2} \sin k(\Omega t-a-\Delta)=h_{1} \sin k(\Omega t-a-\delta),
$$

$h \cos k(\Omega t-a)+h_{2} \cos k(\Omega t-a-\Delta)=h_{1}\left[-\varepsilon \sin k\left(\Omega t-a-\delta^{\prime}\right)+\varepsilon^{\prime} \cos k\left(\Omega t-a-\delta^{\prime}\right)\right]$. En comparant les coefficients de $\sin k(\Omega t-a)$ et $\cos k(\Omega t-a)$, on trouve

$$
\begin{aligned}
& \text { (30.) } \operatorname{tg} k \Delta=\frac{2 \varepsilon}{1-\varepsilon^{2}-\varepsilon^{\prime 2}}, \\
& \text { (31.) }\left\{\begin{array}{l}
h_{2}=-\gamma h, \\
\gamma=\sqrt{\frac{\left(1-\varepsilon^{\prime}\right)^{2}+\varepsilon^{2}}{\left(1+\varepsilon^{\prime}\right)^{2}+\varepsilon^{2}}} .
\end{array}\right.
\end{aligned}
$$

Supposons d'abord que le premier bout du tuyau soit fermé par un plan fixe, dans ce cas le potentiel du mouvement primitif, produit d'une manière quelconque à l'autre bout, sera

$$
h \cos k(\Omega t+x)
$$


après la réflexion produite par le plan fixe $(x=0)$ il deviendra

$$
h \cos k(\Omega t-x) \text {, }
$$

or le potentiel de la somme de ces deux mouvements sera

$2 h \cos k \Omega t \cos k x$.

Lorsque les réflexions de la première onde se sont répétées $n$ fois au bout ouvert, le potentiel sera

$2 h \cos k x\left[\cos k \Omega t-\gamma \cos k(\Omega t-2 a-\Delta)+\gamma^{2} \cos k(\Omega t-4 a-2 \Delta)-\cdots\right.$

$$
\left.+(-\gamma)^{n} \cos k(\Omega t-n(2 a+\Delta))\right] \text {. }
$$

Pour $n=\infty$ cette expression converge vers la limite

$$
\text { (32.) } 2 \frac{h}{\rho} \cos k x \cos (k \Omega t+\theta),
$$

où l'on a posé

$$
\varrho=\sqrt{1+\gamma^{2}+2 \gamma \cos k(2 a+\Delta)}, \quad \sin \theta=\frac{\gamma}{\rho} \sin k(2 a+\Delta) .
$$

Cette valeur du potentiel aura son maximum pour

$$
k(2 a+\Delta)=(2 p+1) \pi,
$$

$p$ étant un nombre entier. En désignant par $\lambda=\frac{2 \pi}{k}$ la longueur d'une onde entière, le mouvement vibratoire aura donc sa plus grande force, quand on a

$$
\text { (33.) } \quad \lambda=\frac{4 a+2 \Delta}{2 p+1} \text { ou } a+\frac{\Delta}{2}=\frac{\lambda}{4}, \frac{3 \lambda}{4}, \frac{5 \lambda}{4} \ldots
$$

Dans ce cas la valeur du potentiel (32.) devient

$$
\text { (34.) } 2 \frac{h}{1-\gamma} \cos k x \cos k \Omega t \text {. }
$$

Supposons en second lieu que les deux bouts du tuyau soient ouverts, et que le mouvement se produise au second bout $(x=a)$, le potentiel du mouvement primitif sera

$$
h \cos k(\Omega t+x) \text {. }
$$

Après la réflexion qui a lieu au premier bout ouvert $(x=0)$, il devient

$$
-h \gamma \cos k(\Omega t-x-\Delta)
$$

le potentiel de la somme de ces deux mouvements sera donc

$$
\boldsymbol{h}[\cos k(\Omega t+x)-\gamma \cos k(\Omega t-x-\Delta)] \text {. }
$$

Lorsque les réflexions se sont répétées un nombre infini de fois, le potentiel sera

$$
\frac{h}{\rho}[\cos k(\Omega t+x-\theta)-\gamma \cos k(\Omega t-x-\Delta-\theta)]
$$

où l'on a posé

$$
\rho=\sqrt{1+\gamma^{4}-2 \gamma^{2} \cos 2 k(a+\Delta)}, \quad \sin \theta=\frac{\gamma^{2}}{\rho} \sin 2 k(a+\Delta) .
$$

Journal für Mathematik Bd. LVIII. Heft 4. 
Le mouvement aura donc sa plus grande force, quand on a

d'où

$$
2 k(\boldsymbol{l}+\Delta)=2 p \pi
$$

(36.) $\quad \lambda=\frac{2(a+\Delta)}{p}$ ou $a+\Delta=\frac{\lambda}{2}, \frac{2 \lambda}{2}, \frac{3 \lambda}{2} \ldots$

Dans ce cas la valeur du potentiel (35.) devient

$$
\text { (37.) }-\frac{h}{1-\gamma} \sin k \Omega t \sin k x+\frac{h}{1+\gamma} \cos k \Omega \iota \cos k x \text {. }
$$

On voit par la valeur (32.) du potentiel que dans le cas où l'un des bouts du tuyau est fermé, il y aura des noeuds ou des points parfaitement immobiles, savoir ceux qui donnent $\sin k x=0$, tandis qu'au contraire dans le cas (37.) où les deux bouts sont ouverts, on ne pourra dire que d'une manière approximative qu'il $\mathrm{y}$ a des points immobiles. Pour ces points on a $\cos k x=0$.

Si le tuyau est cylindrique, $\boldsymbol{R}$ étant le rayon de la section circulaire normale à l'axe, on trouvera

$$
\begin{aligned}
& \varepsilon=\sum_{n=0}^{n=\infty}(\boldsymbol{k R})^{2 n+1} \frac{(-1)^{n}}{\Gamma\left(n+\frac{8}{2}\right) \Gamma\left(n+\frac{5}{2}\right)}, \\
& \varepsilon^{\prime}=\sum_{n=0}^{n=\infty}(\boldsymbol{k R})^{2 n+2} \frac{(-1)^{n}}{\Gamma(n+2) \Gamma(n+3)} .
\end{aligned}
$$

Pour des valeurs très petites de $\boldsymbol{k} \boldsymbol{R}$ on aura

$$
\varepsilon=\frac{8}{3 \pi} k \boldsymbol{R}, \quad \varepsilon^{\prime}=0,
$$

d'où par l'équation (30.)

$$
\Delta=\frac{16}{3 \pi} \boldsymbol{R} \text {. }
$$

Pour un tuyau ouvert aux deux bouts on aura donc

$$
\frac{\frac{1}{2} \lambda p-a}{2 R}=\frac{8}{3 \pi}=0,8488 \text {. }
$$

Ce résultat est parfaitement confirmé par les expériences de M. Zaminer (Anń. de Pogg. 97), qui pour un tuyau ouvert aux deux bouts, d'une longueur de $500^{\text {min }}$ et d'un diamètre $(d)$ de $25^{\text {mm }}$, a trouvé

$$
\frac{1}{2} \lambda=522,2, \quad \text { d'où } \quad \frac{\frac{1}{2} \lambda-a}{d}=0,848 .
$$

Dans le tableau suivant j'ai calculé la valeur de $\frac{\Delta}{d}$ pour différentes valeurs de $2 R$ ou $d$, divisé par $\frac{1}{2} \lambda$. 


\begin{tabular}{c|c||c|c}
$\frac{2 d}{\lambda}$ & $\frac{\Delta}{d}$ & $\frac{2 d}{\lambda}$ & $\frac{\Delta}{d}$ \\
\hline 0,00 & 0,8488 & 0,40 & 0,7293 \\
0,05 & 0,8462 & 0,45 & 0,7080 \\
0,10 & 0,8385 & 0,50 & 0,6873 \\
0,15 & 0,8263 & 0,55 & 0,6672 \\
0,20 & 0,8106 & 0,60 & 0,6480 \\
0,25 & 0,7921 & 0,65 & 0,6298 \\
0,30 & 0,7720 & 0,70 & 0,6125 \\
0,35 & 0,7508 & 0,75 & 0,5962
\end{tabular}

Si l'on applique les chiffres de ce tableau aux données de l'expérience que nous venons de citer, on aura plus exactement: $\frac{\frac{1}{2} \lambda-a}{d}=0,8463$.

Les expériences de M. Werlheim (Ann. de chim. et de phys. 31) donnent, pour les tuyaux cylindriques ouverts aux deux bouts, $\frac{\Delta}{d}=2 \sqrt{ } \cdot 0,187=0,663$, indépendamment du diamètre, tandis que M. Zaminer trouve, que la valeur de $\frac{\Delta}{d}$ diminue, quand le diamètre devient plus grand. Ses expériences donnent cependant un décroissement plus fort, que ne le donne le calcul, dont les résultats sont par conséquent compris entre ceux de ces deux physiciens. Les expériences faites sur des tuyaux fermés ne s'accordent pas avec les résultats du calcul, sans doute parceque le fond du tuyau n'est pas complètement immobile.

Il y a encore beaucoup d'autres problèmes, dont la solution serait importante, mais on ne serait pas à même de contrôler les résultats du calcul au moyen d'expériences. La difficulté que l'on éprouve dans les expériences de s'approcher des suppositions mathématiques, a empêché jusqu'à présent un accord suffisant entre leurs résultats.

\section{5.}

Equilibre du prisme rectangulaire.

Considérons un corps homogène, à élasticité constante, qui soit limité par les trois plans coordonnés, et par trois autres plans parallèles aux premiers, dans les distances $a, b$ et $c$. Si les données relatives aux déplacements des points situés dans les six faces ou aux forces extérieures, qui agissent sur ces faces suffisent pour déterminer complètement l'équilibre intérieur, il faudra résoudre le problème de déterminer par les données tous les déplacements et 
toutes les forces élastiques qui se produisent dans chaque point du corps. Nous allons nous borner ici aux deux cas, qui permettent une solution exacte. Dans le premier cas les déplacements normaux et les forces tangentielles, dans le second les déplacements tangentiels et les forces normales sont donnés, pour les points situés dans les six faces. - On admet que les valeurs des forces données sont compatibles avec les conditions qu'exige l'équilibre extérieur du corps.

Posons

$$
\left\{\begin{array}{l}
\mathrm{F}=-\frac{1}{2 \pi} \Sigma \Sigma \Sigma \int_{0}^{b} d \beta \int_{0}^{c} d \gamma\left[\frac{f(\beta, \gamma)}{r}-\frac{f_{1}(\beta, \gamma)}{r_{1}}\right], \\
r=\sqrt{(x+2 i a)^{2}+\left(y+2 i_{1} b \pm \beta\right)^{2}+\left(z+2 i_{2} c \pm \gamma\right)^{2}}, \\
r_{1}=\sqrt{(x+(2 i+1) a)^{2}+\left(y+2 i_{1} b \pm \beta\right)^{2}+\left(z+2 i_{2} c \pm \gamma\right)^{2}},
\end{array}\right.
$$

où le signe \pm a la même signification qu'auparavant, et où la sommation se rapporte aux trois nombres $i, i_{1}, i_{2}$ dont chacun prend toutes les valeurs entières, positives et négatives, de $-\infty$ jusqu'à $+\infty$.

Il est évident par ce qui précède que la fonction $F$ vérifie les équations suivantes :

$$
\begin{array}{rlrl}
\triangle^{2} \mathrm{~F} & =0, & & \\
{\left[\frac{d \mathrm{~F}}{d x}\right]^{x=0}} & =f(y, z), & & {\left[\frac{d \mathrm{~F}}{d x}\right]^{x=a}=f_{1}(y, z),} \\
{\left[\frac{d \mathrm{~F}}{d y}\right]^{\gamma=0, b}=0,} & & {\left[\frac{d \mathrm{~F}}{d z}\right]^{z=0, c}=0 .}
\end{array}
$$

Introduisons encore, en conservant les mêmes notations, la fonction

(39.) $\quad \mathrm{F}_{2}=-\frac{1}{2 \pi} \Sigma \Sigma \Sigma \int_{0}^{b} d \beta \int_{0}^{c} d \gamma\left[\frac{r}{2} f(\beta, \gamma)-\frac{r_{1}}{2} f_{1}(\beta, \gamma)\right]$,

elle satisfera aux équations

$$
\begin{aligned}
\Delta^{2} \mathrm{~F}_{2} & =\mathrm{F}, \\
{\left[\frac{d \mathrm{~F}_{2}}{d y}\right]^{y=0, b} } & =0, \quad\left[\frac{d \mathrm{~F}_{2}}{d z}\right]^{z=0, c}=0 ;
\end{aligned}
$$

et l'on trouvera en outre

$$
\begin{array}{rlrl} 
& {\left[\frac{d^{3} \mathrm{~F}_{2}}{d x^{3}}\right]^{x=0}=f(y, z),} & {\left[\frac{d^{3} \mathrm{~F}_{2}}{d x^{3}}\right]^{x=a}=f_{1}(y, z),} \\
\frac{d^{2}}{d y^{2}}\left[\frac{d \mathrm{~F}_{2}}{d x}\right]^{x=0, a} & =0, & \frac{d^{2}}{d z^{2}}\left[\frac{d \mathrm{~F}_{2}}{d x}\right]^{x=0, a} & =0 .
\end{array}
$$

Bien que les expressions $F$ et $F_{2}$ aient des valeurs infinies, cette circonstance 
n'infirmera en rien l'exactitude des développements ultérieurs, car on ne se servira que de celles des dérivées de ces expressions qui restent finies.

Si l'on transforme la valeur de $\frac{d \mathrm{~F}}{d x}$, soit en remplaçant la triple somme par une intégrale triple, soit en développant $f(\beta, \gamma)$ et $f_{1}(\beta, \gamma)$ suivant les cosinus des multiples de l'arc $\frac{\pi \beta}{b}$ et de l'arc $\frac{\pi \gamma}{c}$, on parviendra à une autre expression, dont l'intégrale par rapport à $x$ est

où

$$
\left\{\begin{aligned}
\mathrm{F}=- & \frac{1}{b c} \Sigma \Sigma \int_{0}^{b} d \beta \int_{0}^{c} d \gamma \frac{\cos \frac{\pi i_{1} y}{b} \cos \frac{\pi i_{2} z}{c} \cos \frac{\pi i_{1} \beta}{b} \cos \frac{\pi i_{2} \gamma}{c}}{p\left(e^{p a}-e^{-p a}\right)} \\
& \times\left[\left(e^{p(a-x)}+e^{-p(a-x)}\right) f(\beta, \gamma)-\left(e^{p x}+e^{-p x}\right) f_{1}(\beta, \gamma)\right]
\end{aligned}\right.
$$

$$
p=\pi \sqrt{\frac{i_{1}^{2}}{b^{2}}+\frac{i_{2}^{2}}{c^{2}}}
$$

Cette valeur de F qui est finie, satisfait aux mêmes conditions que l'on vient d'écrire pour la valeur (38.) de $F$. On trouve pour $F_{2}$ une valeur correspondante qui est finie, savoir:

$$
\begin{aligned}
\mathbf{F}_{2}= & -\frac{1}{b c} \Sigma \Sigma \int_{0}^{b} d \beta \int_{0}^{c} d \gamma \frac{\cos \frac{\pi i_{1} y}{b} \cos \frac{\pi i_{2} z}{c} \cos \frac{\pi i_{1} \beta}{b} \cos \frac{\pi i_{2} \gamma}{c}}{2 p^{2}\left(e^{p a}-e^{-p a}\right)} \\
\times & {\left[\begin{array}{l}
\left((a-x)\left(e^{p(a-x)}-e^{-p(a-x)}\right)-\left(a \frac{e^{p a}+e^{-p a}}{e^{p a}-e^{-p a}}+\frac{1}{p}\right)\left(e^{p(a-x)}+e^{-p(a-x)}\right)\right) f(\beta, \gamma) \\
-\left(x\left(e^{p x}-e^{-p x}\right)-\left(a \frac{e^{p a}+e^{-p a}}{e^{p a}-e^{-p a}}+\frac{1}{p}\right)\left(e^{p x}+e^{-p x}\right)\right) f_{1}(\beta, \gamma)
\end{array}\right] }
\end{aligned}
$$

Introduisons en outre les fonctions $\mathfrak{F}$ et $\Phi$ en les faisant respectivement dépendre de $f, f_{1}$ et de $\varphi, \varphi_{1}$ de la même manière que $F$ dépend de $f, f_{1}$. Introduisons enfin les fonctions $\mathfrak{F}^{\prime}$ el $\Phi^{\prime}$, qui dépendront des mêmes fonctions $\mathfrak{f}_{,} \mathfrak{f}_{1}$ et $\varphi, \varphi_{1}$ et qui ne différeront des expressions de $\mathfrak{F}$ et $\Phi$ contenues dans les équations analogues à (38.) que par l'acceptation du signe \pm . Nous distinguerons le nouveau sens qu'il faut attribuer à ce signe dans les sommes où il se trouve, en l'écrivant $[ \pm]$, et en $y$ attachant la signification définie par l'équation

$$
\text { fonct. }([ \pm])=\text { fonct. }(+)-\text { fonct. }(-) \text {. }
$$

Il y a des valeurs finies de $F^{\prime}, \Phi^{\prime}$ semblables aux valeurs de $\mathfrak{F}$, $\Phi$ qui sont contenues dans les équations analogues à (40.). On arrive à ces nouvelles valeurs en remplaçant dans $\mathfrak{F}, \Phi$ tous les cosinus par des sinus. On voit 
aisément que l'on aura

$$
\begin{array}{rlrl}
\mathfrak{F}^{\prime}=0 \text { et } \Phi^{\prime}=0 \text { pour } y=0, y=b, z=0, z=c, \text { et } \\
{\left[\frac{d \mathfrak{F}^{\prime}}{d x}\right]^{x=0}=f(y, z),} & {\left[\frac{d \mathfrak{F}^{\prime}}{d x}\right]^{x=a}=\mathfrak{f}_{1}(y, z),} \\
{\left[\frac{d \Phi^{\prime}}{d x}\right]^{x=0}=\varphi(y, z),} & {\left[\frac{d \Phi^{\prime}}{d x}\right]^{x=\alpha}=\varphi_{1}(y, z) .}
\end{array}
$$

Posons maintenant

où

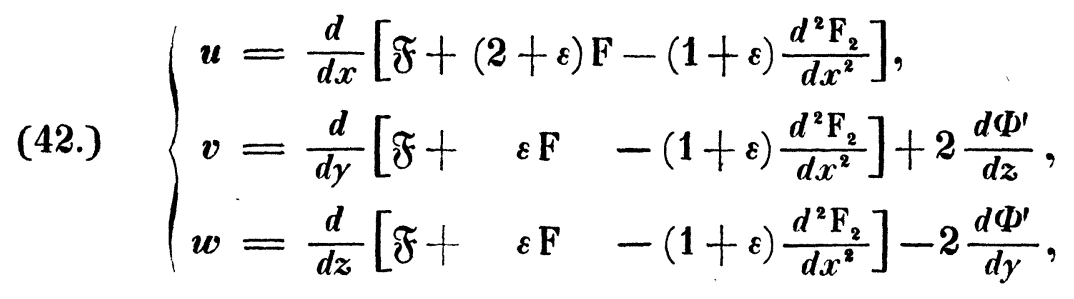

$$
\varepsilon=\frac{\lambda}{\lambda+2 \mu}
$$

Ces valeurs des composantes, qui satisfont aux équations de l'équilibre, dónnent

$$
\begin{array}{rlrl}
{[u]^{x=0}} & =f(y, z)+f(y, z), & {[u]^{x=a}=f_{1}(y, z)+f_{1}(y, z),} \\
{\left[\frac{1}{2 \mu} T_{2}\right]^{x=0}=\frac{d f(y, z)}{d z}-\frac{d \varphi(y, z)}{d y},} & {\left[\frac{1}{2 \mu} T_{2}\right]^{x=a}=\frac{d f_{1}(y, z)}{d z}-\frac{d \varphi_{1}(y, z)}{d y},} \\
{\left[\frac{1}{2 \mu} T_{3}\right]^{x=0}=\frac{d f(y, z)}{d y}+\frac{d \varphi(y, z)}{d z},} & {\left[\frac{1}{2 \mu} T_{3}\right]^{x=a}=\frac{d f_{1}(y, z)}{d y}+\frac{d \varphi_{1}(y, z)}{d z},}
\end{array}
$$

tandis que les déplacements normaux et les forces tangentielles qui se rapportent aux points situés dans les autres faces du prisme sont nuls. Les six fonctions $f, f$ etc. seront donc déterminées par les dernières six équations, et, si l'on connaît les déplacements normaux et les forces tangentielles qui se rapportent aux points situés dans les deux faces $(x=0)$ et $(x=a)$, les composantes des déplacements qui en dépendent, seront déterminées par les équations (42.) De la même manière on trouvera les composantes des déplacements produits par des déplacements normaux et des forces tangentielles qui se rapportent aux points situés dans les autres faces du prisme. Il est bon de remarquer, que la solution que l'on vient de donner contient des fonctions arbitraires; elles $y$ entrent par la détermination de $f(y, z), f_{1}(y, z)$, $\varphi(y, z)$ et $\varphi_{1}(y, z)$.

Lorsqu'il s'agit de résoudre le problème inverse dans lequel les forces normales et les déplacements tangentiels pour les points situés dans les six faces sont donnés, on formera les composantes des déplacements qui dépendent 
des forces normales et des déplacements tangentiels relatifs aux points situés dans les deux faces $(x=0)$ et $(x=a)$; elles sont données par les équations:

$$
\text { (43.) }\left\{\begin{aligned}
u & =\frac{d^{2}}{d y d z}\left[2 \mathrm{~F}-(1+\varepsilon) \frac{d^{2} \mathrm{~F}_{2}}{d x^{2}}\right]+\frac{d^{2} \mathfrak{F}^{\prime}}{d x^{2}}, \\
v & =\frac{d^{2}}{d x d z}\left[\Phi-(1+\varepsilon) \frac{d^{2} \mathrm{~F}_{2}}{d y^{2}}\right]+\frac{d^{2} \mathscr{F}^{\prime}}{d x d y}, \\
w & =\frac{d^{2}}{d x d y}\left[-\Phi-(1+\varepsilon) \frac{d^{2} \mathrm{~F}_{2}}{d z^{2}}\right]+\frac{d^{2} \mathfrak{F}^{\prime}}{d x d z} .
\end{aligned}\right.
$$

Ces valeurs des composantes, qui satisfont aux équations de l'équilibre, donnent

$$
\begin{aligned}
{\left[\frac{1}{2 \mu} N_{1}\right]^{x=0} } & =\frac{d^{2} f(y, z)}{d y}-\left(\frac{d^{2}}{d y^{2}}+\frac{d^{2}}{d z^{2}}\right) \mathfrak{f}(y, z), \\
{\left[\frac{1}{2 \mu} N_{1}\right]^{x=a} } & =\frac{d^{2} f_{1}(y, z)}{d y d z}-\left(\frac{d^{2}}{d y^{2}}+\frac{d^{2}}{d z^{2}}\right) \mathfrak{f}_{1}(y, z), \\
{[v]^{x=0} } & =\frac{d f(y, z)}{d y}+\frac{d \varphi(y, z)}{d z} \\
{[v]^{x=a} } & =\frac{d f_{1}(y, z)}{d y}+\frac{d \varphi_{1}(y, z)}{d z} \\
{[w]^{x=0} } & =\frac{d \mathfrak{f}(y, z)}{d z}-\frac{d \varphi(y, z)}{d y} \\
{[w]^{x=a} } & =\frac{d f_{1}(y, z)}{d z}-\frac{d \varphi_{1}(y, z)}{d y}
\end{aligned}
$$

tandis que les forces normales et les déplacements tangentiels qui se rapportent aux points situés dans les autres faces sont nuls. On pourra donc déterminer les fonctions $f, f$ etc. par les dernières six équations et le problème sera résolu.

Copenhague, 28 novembre 1860. 